\section{A review of the formulas for the standard error of a threshold from the method of constant stimuli}

\section{SERGIO CESARE MASIN and GIULIO VIDOTTO University of Padua, Padua, Italy}

\section{OVERVIEW OF METHOD}

The threshold of a given perceptual variable, $\psi$, is the value of the associated physical variable, $\phi$, corresponding to the value of $\psi$ having a probability, $\mathrm{p}$, of being detected equal to .5 . To find the threshold, the observer is shown the so-called variable stimuli (VSs) in random order, one after the other. The VSs are physical objects in which generally only one variable is varied, for example, the frequency of a sound, the weight of an object, etc. As a rule, $n$ values, $\phi_{1}, \phi_{2}, \ldots, \phi_{n}$, of such a variable are chosen so that $\phi_{2}-\phi_{1}=\phi_{3}-\phi_{2}=\ldots=\phi_{n}-\phi_{n-1}$ and $\phi_{i}<\phi_{i+1}$. As a recommendation, the probability of detecting the values of $\psi$ corresponding to $\phi_{1}$ and $\phi_{n}$ ought to be greater than 0 and less than 1 , respectively. The observer is asked to report whether he detects the particular values of the perceptual variable $\psi$ associated with $\phi_{1}, \phi_{2}, \ldots$, or $\phi_{n}$. The number of times, $N^{*}$, the observer detects $\psi$ is recorded for each VS. If $N^{*}$ is divided by $\mathrm{N}$-the number of times a single VS is shown to the observer-an estimate, $p$, of the probability of detecting $\psi$ is obtained.

Given the $p$ values, the problem then arises of finding the best estimate of $\phi$ corresponding to $p=.5$, that is, of the threshold. Fechner $(1860$, chap. 7$)$ first suggested the conversion of the probabilities $\mathrm{p}$ to $\mathrm{z}$ scores, and Müller (1879) seems to be the first who fitted the normal integral to the probabilities $p$. If the distribution of the $p$ values on $\phi$ is normal (this assumption is known as the phi-gamma hypothesis), then the regression of the $\mathrm{z}^{*}$ scores on $\phi$ is linear; that is,

$$
z=a+b \phi
$$

Since $p=.5$ corresponds to $z=0$, the threshold is

$$
\phi_{L}=-\frac{a}{b}
$$

To compute the limen, it is sufficient to estimate the values of the coefficients $a$ and $b$ by the common statistical methods used for fitting a line.

Correspondence may be directed to either author at Istituto di Psicologia Sperimentale, University of Padua, Piazza Capitaniato, 35100 Padua, Italy.
When the weighted least squares method is used, $a$ and $b$ are estimated by the following equations:

$$
\begin{gathered}
a=\frac{\Sigma W z \Sigma W \phi^{2}-\Sigma W \phi \Sigma W \phi z}{\Sigma W \Sigma W \phi^{2}-(\Sigma W \phi)^{2}}, \\
b=\frac{\Sigma W \Sigma W \phi z-\Sigma W z \Sigma W \phi}{\Sigma W \Sigma W \phi^{2}-(\Sigma W \phi)^{2}} \\
W=e^{-z^{2}} \frac{1}{4 p(1-p)}
\end{gathered}
$$

In case of the unweighted least squares method ${ }^{1}$ being used, $a$ and $b$ are obtained from Equations 3 and 4 by putting $W=1$. For the reason underlying the use of W, see Brown and Thomson (1940, pp. 67-69).

\section{REVIEW OF THE FORMULAS WHEN THE PHI-GAMMA HYPOTHESIS IS ADOPTED}

Urban (1910, p. 226) first proposed a formula for the SE of a threshold based on linear regression analysis. It reads as follows:

$$
s_{L}=s\left[\frac{\Sigma W\left(z^{*}-z\right)^{2}}{n-2} \cdot \frac{\Sigma W \phi^{2}+\left(\frac{a}{b}\right)^{2} \Sigma W}{\Sigma W \Sigma W \phi^{2}-(\Sigma W \phi)^{2}}\right]^{1 / 2},
$$

where $\phi, p$, and $z^{*}$ are as already defined, $a$ and $b$ are the coefficients given by Equations 3 and $4, z$ is the $\mathrm{z}$ score estimated by Equation 1. The symbols $\mathrm{n}$ and $\mathrm{s}$ represent, respectively, the number of VSs and the standard deviation of the distribution of $\mathrm{ps}$, which is equal to $1 / \mathrm{b}$.

According to Thomson (1914), this formula is inapplicable, because Urban did not consider that weights $W$ were a function of $p$ and assumed $a$ and $b$ were stochastically independent.

Thomson (1914, p. 50) developed another, more complicated, formula, again based on linear regression analysis. It reads as follows:

$$
\begin{gathered}
s_{L}=\frac{1}{D \sqrt{N}} \sqrt{\Sigma[\mu(z \alpha+\beta)+\xi \alpha]^{2}}, \\
D=\Sigma W \Sigma W \phi z-\Sigma W \phi \Sigma W z, \\
\mu=\frac{W(2 p-1)}{\sqrt{p(1-p)}}-z \sqrt{2 \pi W}, \\
\alpha=\phi \Sigma W \phi-\frac{a}{b} \Sigma W \phi-\Sigma W \phi^{2}+\frac{a}{b} \phi \Sigma W,
\end{gathered}
$$




$$
\begin{aligned}
& \beta=\phi \Sigma W \phi z-\phi^{2} \Sigma W z+\frac{a}{b} \Sigma W \phi z-\frac{a}{b} \phi \Sigma W z, \\
& \xi=\sqrt{\pi W / 2}
\end{aligned}
$$

where $\mathbf{N}$ is the number of times each VS is presented to the observer. It is assumed that $\mathbf{N}$ is the same for each VS.

Boring $(1916$, p. 317) used the following formula for the SE, which he considered to correspond statistically to the "SE of a mean":

$$
s_{\mathrm{L}}=\frac{\mathrm{s}}{\sqrt{\Sigma N W}}
$$

In an attempt to achieve a formula simpler than Thomson's, Culler (1927, p. 465) reproposed Boring's formula, using a different mode of weighting:

$$
\begin{gathered}
s_{L}=\frac{s}{\sqrt{\Sigma N U p}}, \\
U=2 W / \pi .
\end{gathered}
$$

Linder (1935) compared the formulas by Boring, Culler, and Thomson through a random sampling experiment, although his usage of Boring's and Culler's formulas was inappropriate because, in entering the formulas with the values taken from the theoretical psychometric function, he put $h$ equal to one instead of $s$. Since $s=1 / h \sqrt{2}$, this entails the introduction in the denominator of the square root of 2 . In the light of Linder's results, but unaware of this fact, Guilford (1954, p. 132) adopted Culler's formula using $W$ in lieu of $U$; that is,

$$
s_{L}=\frac{s}{\sqrt{\Sigma N W p}}
$$

Another formula based on linear regression analysis was suggested by Gaddum (1933). See, also, Finney (1962, pp. 246-254) and Bock and Jones (1968, pp. 33-41) for the theoretical basis. It reads as follows:

$$
\begin{gathered}
s_{L}=s\left[\frac{1}{\Sigma N U}+\frac{\left(-\frac{a}{b}-\bar{\phi}\right)^{2}}{\Sigma N U(\phi-\bar{\phi})^{2}}\right]^{1 / 2}, \\
\bar{\phi}=\frac{\sum N U \phi}{\Sigma N U} .
\end{gathered}
$$

Both Thomson and Gaddum attained their formulas from Equation 2 by the same customary basic procedure (cf. Kendall \& Stuart, 1958, pp. 231-232). To obtain an approximate estimate of the SE,
Thomson started directly from the variances of $\mathrm{ps,}$ while Gaddum considered the variances of $a$ and $b$. However, since the starting point is Equation 2 in both cases, the two formulas must give the same theoretical value.

Still later, Woodworth (1938, p.414; cf. Woodworth \& Schlosberg, 1955, p. 208) suggested another formula. Spearman (1908) showed that when we uncumulate the distribution of $p s$ we end up with a normal distribution, which is interpreted as a distribution of probabilities of momentary limina. It may easily be shown that the number of these limina is $\mathbf{N}$, which is also the number of presentations of one VS to the observer. Since the distribution of momentary limina has been derived from the psychometric function, it follows that the psychometric function (which is a cumulative distribution of the probability of the response "I detect $\psi$ ") is also a cumulated distribution of the probabilities of momentary limina. It is most probably this fact which led Woodworth to suggest the customary formula for the SE of a mean as an estimate of the SE of the threshold; that is,

$$
\mathrm{s}_{\mathrm{L}}=\frac{\mathrm{s}}{\sqrt{\mathrm{N}-1}}
$$

\section{EVALUATION OF THE FORMULAS THROUGH A RANDOM SAMPLING EXPERIMENT}

Since a number of formulas have been proposed for the SE of the threshold, it is necessary to determine the best estimator. This problem may be solved through a random sampling experiment.

The SE of the threshold is the standard deviation of the sampling distribution of the thresholds. It will be assumed that the distribution of probabilities of response on the stimulus values, $\phi$, is normal (phigamma hypothesis). Essentially, the random sampling experiment consists in taking the probabilities from the normal curve and in simulating subject's responses through a computer on the basis of these theoretical probabilities.

\section{Procedure}

First, the range of stimulus values is chosen. Since the unit of measurement is arbitrary, $z$ scores were chosen. The ranges were: $(-2.5,2.5),(-2,2),(-1.5,1.5),(-1,1)$, and $(-.5, .5)$. These were concentric ranges, which we define $(u, v)$. From them, eccentric ranges were also obtained by subtracting iteratively .5 from $v$. This process was stopped when $v=0$. An IBM 370/158 computer was then programmed to carry out the following operations ${ }^{2}$ :

(1) Each stimulus range was subdivided in $n-1$ equal intervals, where $n$ is the number of stimulus values. All integers from 4 to 11 were substituted for $n$. This operation generates the $\phi$-values, that is, the theoretical stimulus values. For instance, given the range $(-1,1)$ and $n=5$, the $\phi$-values are $-1,-.5,0,+.5$, and +1 .

(2) Subjects' responses were simulated as follows. Since the $\phi$ values are $z$ scores, they may be subsequently transformed into their corresponding theoretical probability from the normal curve. 
A pseudorandom number included in the interval $[0,1]$ was generated. When the number was less than the theoretical probability for a given stimulus value, the computer output "greater"; when the number was greater than the theoretical probability, the computer output "less." This operation was repeated $\mathrm{N}$ times, where $\mathbf{N}$ is the number of responses per stimulus value, which is assumed to be the same for each VS. The responses "greater"' were counted and divided by $\mathrm{N}$ to obtain the observed probabilities. The values $10,20,40,100,200$, and 400 were assigned to $\mathrm{N}$.

(3) The observed probabilities so obtained were transformed into $\mathrm{z}$ scores. The coefficients $\mathrm{a}$ and $\mathrm{b}$ in the regression Equation 1 were estimated by the least squares method. The values $1 / 2 \mathrm{~N}$ and $1-(1 / 2 N)$ were substituted for the observed probabilities of 0 and 1 , respectively. (There are various suggestions as to the value to be substituted for a p of 0 or 1 ; the values we used seem to have the effect of best reducing bias; Bock \& Jones, 1968, p. 22.) Finally the limen was computed by Equation 2 .

(4) Steps 1 to 3 were iterated 100 times; thus, a sampling distribution of 100 limina was obtained. The standard deviation of this distribution, an estimate of the SE of the limen, was computed.

(5) A chi-square test was applied to test whether the SE obtained by the sampling experiment differed statistically from the theoretical value provided by the formulas. The test was applied for all combinations of the ranges of probability and the values of $\mathrm{n}$ and $\mathrm{N}$.

\section{Results and Conclusions}

On the basis of samples of 100 thresholds, the Thomson-Gaddum formula (Equations 7 and 11) and the Culler modified formula (Equation 10) give good estimates of the SE of the threshold, except for the extreme cases in which the probability of detection associated with $\phi_{1}\left(\phi_{n}\right)$ is greater (less) than .5 , $\mathrm{p}<.001$. The formulas by Urban (Equation 6), Culler (Equation 9), and Woodworth (Equation 12) consistently overestimate the SE of the threshold, while Boring's formula (Equation 8) consistently underestimates $(\mathrm{p}<.001)$.

In Figures 1, 2, and 3, the values of the SE computed by the various formulas are plotted against the various theoretic ranges of probabilities for $n=5$ and $\mathrm{N}=100$. Urban's formula does not appear because it overestimates too much. Each dot joined by the

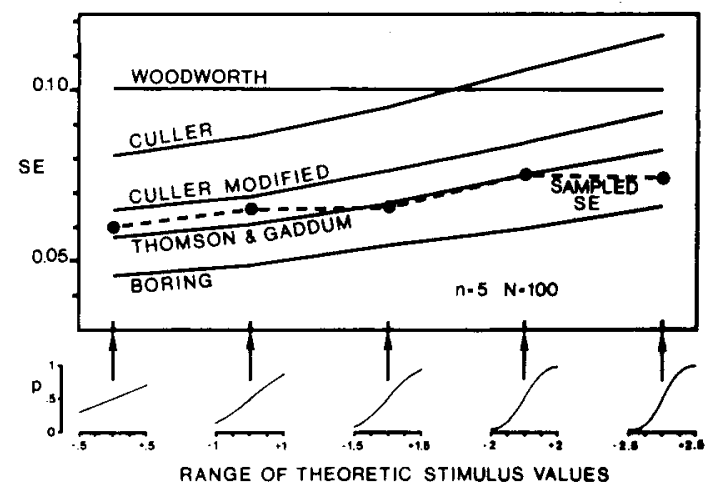

Figure 1. The variation in the $\mathrm{SE}$ of the limen, given by the various formulas (solid lines) and by the sampling experiment (dashed line), as a function of the range of the stimulus values for a concentric psychometric function.

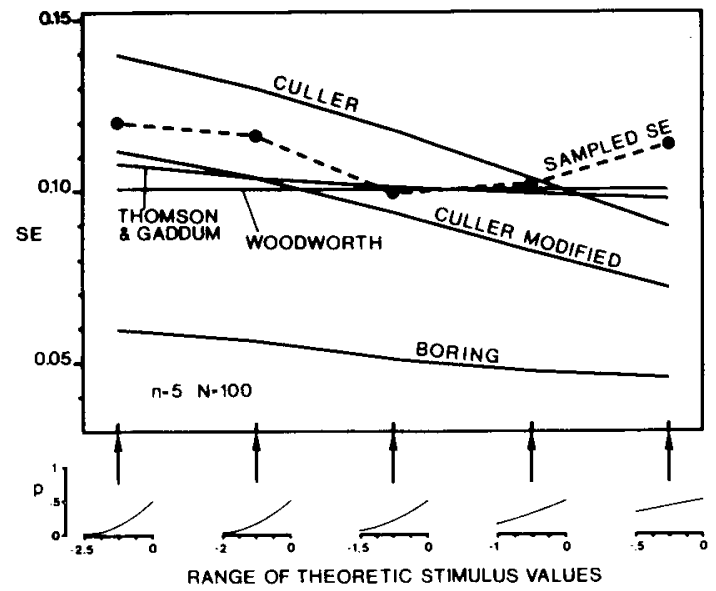

Figure 2. The variation in the SE of the limen, given by the various formulas (solid lines) and by the sampling experiment (dashed line), as a function of the range of the stimulus values for a very eccentric psychometric function.

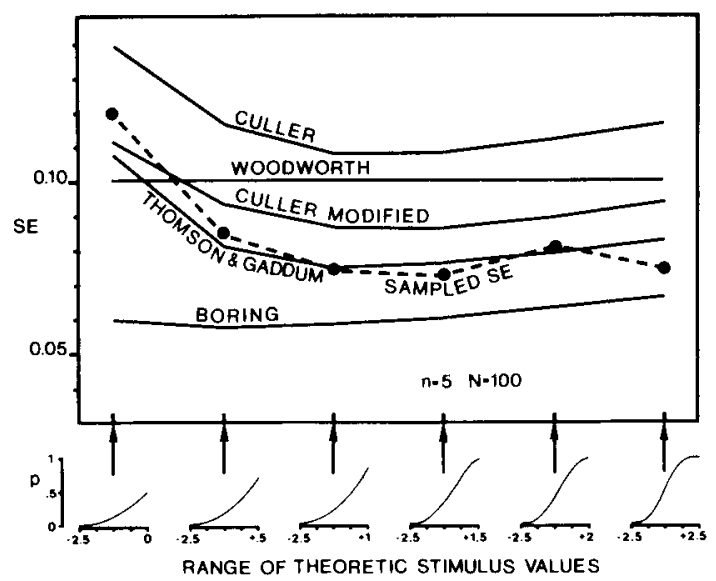

Figure 3. The variation in the SE of the limen, given by the various formulas (solid lines) and by the sampling experiment (dashed line), as a function of combined values both of eccentricity of the psychometric function and of amplitudes of the ranges of stimulus values.

dashed line corresponds to a SE obtained from a sample of 10,000 limina. When, as in these figures, the variability of the sampled SEs is highly reduced, the Thomson-Gaddum formulas come out clearly as the most precise ones.

However, Thomson-Gaddum formulas also misbehave when very eccentric psychometric functions and very small ranges of probabilities are used. The formulas produce a slight underestimation in the extreme cases because they do not take into account the procedure the experimenter is forced to adopt when $\mathrm{p}=0$ or $\mathrm{p}=1$, a procedure that always produces a bias.

A plausible reason for the behavior of Culler's original and modified formulas, and Boring's for- 
mula in Figure 1-their derivation was based on empirical or intuitive grounds to a fair degree-is that they are structurally similar to the first additive part in Gaddum's formula. In fact, the second additive part in this formula contributes very little to the overall SE of the limen when the psychometric function is nearly concentric.

\section{REFERENCES}

Bock, D. R., \& Jones, L. V. The measurement and prediction of judgment and choice. San Francisco: Holden-Day, 1968.

Boring, E. G. The number of observations on which a limen may be based. American Journal of Psychology, 1916, 27, 315-319.

Brown, W., \& Thомson, G. H. The essentials of mental measurement. Cambridge: Cambridge University Press, 1940.

Culler, E. Studies in psychometric theory. XIV. On the probable error of the limen (method of constant stimuli). Journal of Experimental Psychology, 1927, 10, 463-477.

Fechner, G. T. Elemente der Psychophysik. Leipzig: Breitkopf und Härtel, 1860.

Finney, D. J. Probit analysis. Cambridge: Cambridge University Press, 1962.

GADDUM, J. H. Reports on biological standards. III. Methods of biological assay depending on a quantal response. Medical Research Council Special Report Series, London, 1933, Whole No. 183.

Gullford, J. P. Psychometric methods. New York: McGrawHill, 1954.
Kendall, M. G., \& Stuart, A. The advanced theory of statistics (Vol. 1): Distribution theory. London: Griffin, 1958.

Linder, F. E. The accuracy of the constant method. American Journal of Psychology, 1935, 47, 504-508.

Müller, G. E. Über die Massbestimmungen des Ortssinnes der Haut mittels der Methode der richtigen und falschen Fälle. Pflüger's Archiv für die gesamte Physiologie, 1879, 19, 191-235.

Spearman, C. The method of 'right and wrong cases' ('constant stimuli') without Gauss's formulae. British Journal of Psychology, 1908, 2, 227-242.

Thomson, G. H. The accuracy of the $\Phi(\gamma)$ hypothesis. British Journal of Psychology, 1914, 7, 44-55.

UrBan, F. M. Die psychophysischen Massmethoden als Grundlagen empirischer Messungen. Archiv für die gesamte Psychologie, 1909, 15, 261-335; 1910, 16, 168-227.

WoodwoRTH, R. S. Experimental psychology. New York: Holt, 1938.

Woodworth, R. S., \& Schlosberg, H. Experimental psychology. New York: Holt, 1955.

\section{NOTES}

1. All the statements in the text should be understood as applying at the same time to the weighted and unweighted least squares methods, except when otherwise specified.

2. The programs were written in FORTRAN IV. In order to check for their correctness, they were rewritten in PASCAL 8000.

(Manuscript received October 28, 1981; revision accepted for publication February 5, 1982.) 